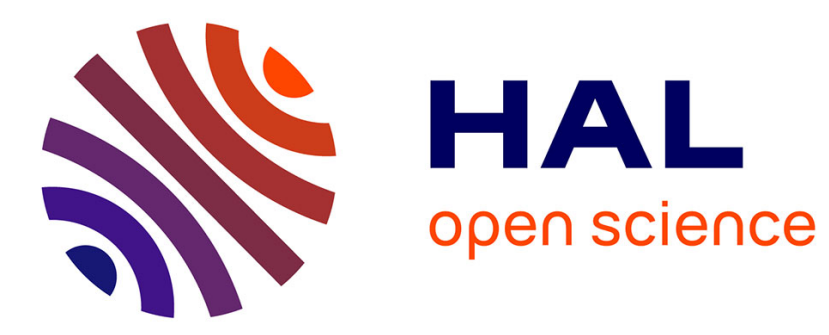

\title{
Bit Error Rate Calculation for a Multiband Non Coherent On-Off Keying Demodulation
}

Frédéric Guilloud, Emmanuel Boutillon, Jean-Luc Danger

\section{To cite this version:}

Frédéric Guilloud, Emmanuel Boutillon, Jean-Luc Danger. Bit Error Rate Calculation for a Multiband Non Coherent On-Off Keying Demodulation. IEEE International Conference On Communications (ICC 2002), Apr 2002, New-York, NY, United States. pp.202-206, 10.1109/ICC.2002.996845 . hal02295908

\section{HAL Id: hal-02295908 \\ https://hal.science/hal-02295908}

Submitted on 24 Sep 2019

HAL is a multi-disciplinary open access archive for the deposit and dissemination of scientific research documents, whether they are published or not. The documents may come from teaching and research institutions in France or abroad, or from public or private research centers.
L'archive ouverte pluridisciplinaire HAL, est destinée au dépôt et à la diffusion de documents scientifiques de niveau recherche, publiés ou non, émanant des établissements d'enseignement et de recherche français ou étrangers, des laboratoires publics ou privés. 


\section{Bit Error Rate Calculation for a Multiband Non Coherent On-Off Keying Demodulation}

\author{
F. Guilloud \\ ENST - Dpt COMELEC \\ 46, rue Barrault - 75013 Paris
}

\author{
E. Boutillon \\ Université de Bretagne Sud - LESTER \\ BP 92116 - 56321 Lorient CEDEX
}

\author{
J.L. Danger \\ ENST - Dpt COMELEC \\ 46, rue Barrault - 75013 Paris
}

\begin{abstract}
The purpose of this paper is to calculate the bit error rate (BER) of a multiband non coherent on-off keying (OOK) demodulation. The results fit perfectly the simulations of the system. It allows us to study the influence of the filter and the decimation factor on the modulation performance. It is also possible to optimize the system, by means of other criteria (e.g. system complexity, jammer sensitivity) thus avoiding time consuming simulations.
\end{abstract}

Index Terms - bit error rate, non-coherent OOK demodulation, quadratic forms, correlation, filtering, chi-square law.

\section{INTRODUCTION}

B IT error rate (BER) specifications are very important in the design of digital telecommunication systems. BER calculations for optimum receivers are common [1], [2]. Yet if the receiver is not optimum, or if the receiver features additional filters, system simulations are often used. The major drawback is that simulations are generally time consuming, thus limiting the ability of the system to be optimized.

The purpose of this paper is first to establish a theoretical mathematical formula for the BER, taking into account the filter bank used in a telemetry non-coherent receiver. The goal is to avoid lengthy simulations when optimizing a multiband demodulator, with regard to jammer sensitivity and system complexity. The filter-characteristics influence on the BER in the case of non-coherent OOK demodulations is studied.

The system is made of a very large number of transducers whose measurements are to be automatically and remotely read by a radio concentrator (Fig. 1). Applications are quite numerous in the metering business; for example extensive breeding of cattle in south America: herd movements have to be studied in order to optimize their feeding.

The transmitters of these various applications have common requirements: they should be very cheap and have a very low power consumption. Therefore a simple modulation scheme must be chosen. We consider in this paper an OOK modulation in association with a bi-phase coding (or Manchester coding), each transmitter having its own frequency carrier. In that kind of modulation, each bit is coded by two chips: ' 10 ' when the

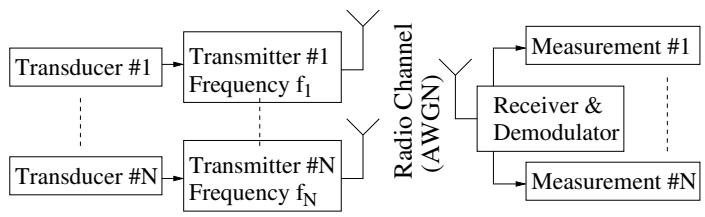

Fig. 1

TELEMETRY SYSTEM
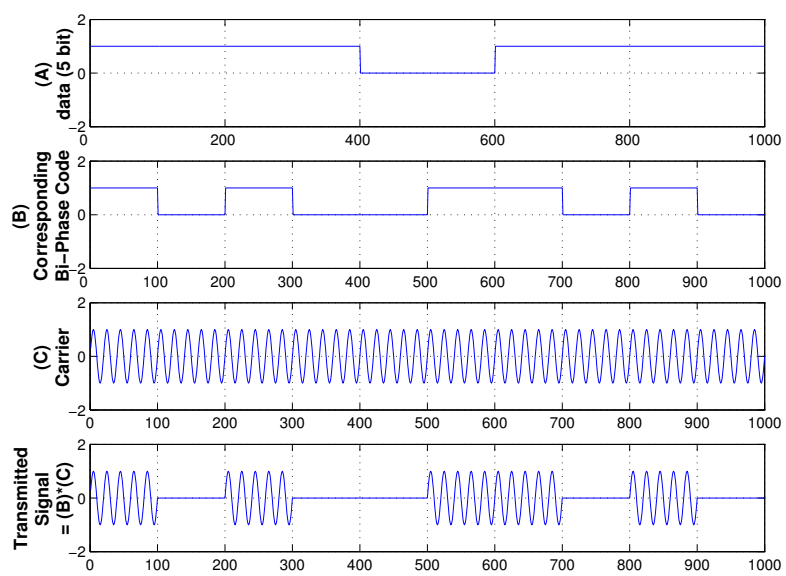

Fig. 2

OOK BIPHASE MODULATION

bit is ' 1 ', and ' 01 ' when the bit is ' 0 '. The chips ' 1 ' and ' 0 ' are often called respectively mark and space (Fig. 2).

We begin this paper in section II with the demodulation principles, setting up the notations and the basic equations. In section III, the whole bit error rate calculation is explained. In section IV, we compare the bit error rates obtained by simulations and by evaluation of our mathematical expressions. Conclusion and future work description are given in section $\mathrm{V}$.

\section{Demodulation PRinciples}

\section{A. Basic Equations}

We consider here a signal modulated as described above. This signal is sent through an additive white gaussian noise channel (AWGN), and is oversampled with $S$ samples per chip, i.e. $2 S$ per bit. The signal is then filtered by a digital FIR filter bank before being demodulated (Fig. 3). The influence of decimation has also to be studied because the demodulator includes a filter bank based solution [3].

Let $x_{p}=a_{p}+b_{p}$ be the received sample number $p$, where $a_{p}$ is the magnitude of the OOK signal before the AWGN channel; thus $a_{p}=0$ for space chips and $a_{p}=U>0$ for mark chips. $b_{p}$ stands for the AWGN samples. Let also $\sigma^{2}$ be the variance of the AWGN.

We now focus our attention on the filtering of a bit. Let the column vector $H=\left(h_{n}\right)_{n \in\{N, \ldots, 1\}}$ represent the filter coefficients. Let the sample $x_{n+i \Delta}$ be noted with the double index $x_{n, i}$, where $n \in\{1, \ldots, N\}, i \in\left\{0, \ldots, 2 S_{f}-1\right\}$. Let $S_{f}$ be the number of samples in a filtered chip after decimation. In fact, 


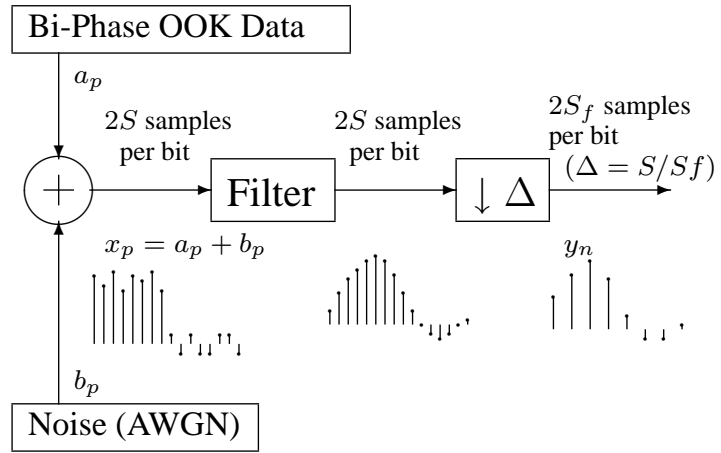

Fig. 3

DigITAL SIGNAL PROCESSING MODEL BEFORE BIT DECODING

the convolution is done each $\Delta T$ in order to take into account the decimation at the same time, $T$ being the sample period: so $\Delta=S / S_{f}$.

The transposition of a matrix $M$ will be denoted by $M^{\prime}$. Using the notations:

$$
\begin{aligned}
A_{i} & =\left(a_{1, i} \cdots a_{n, i} \cdots a_{N, i}\right)^{\prime} \\
B_{i} & =\left(b_{1, i} \cdots b_{n, i} \cdots b_{N, i}\right)^{\prime} \\
H & =\left(h_{N} \cdots h_{n} \cdots h_{1}\right)^{\prime}
\end{aligned}
$$

with $X_{i}=A_{i}+B_{i}$, we obtain:

$$
\begin{array}{lll}
A^{(1)}=\left(A_{1} \cdots A_{S_{f}}\right) & , & A^{(2)}=\left(A_{S_{f}+1} \cdots A_{2 S_{f}}\right) \\
B^{(1)}=\left(B_{1} \cdots B_{S_{f}}\right) & , & B^{(2)}=\left(B_{S_{f}+1} \cdots B_{2 S_{f}}\right) \\
X^{(1)}=A^{(1)}+B^{(1)} & , & X^{(2)}=A^{(2)}+B^{(2)}
\end{array}
$$

where index ${ }^{(1)}$ et ${ }^{(2)}$ denote respectively the first and the second chip of the bit.

The filtering over the first and the second chip is the matrix product:

$$
\begin{aligned}
Y^{(1)} & =X^{(1)^{\prime}} H=\left(y_{1} \cdots y_{i} \cdots y_{S_{f}}\right)^{\prime} \\
Y^{(2)} & =X^{(2)^{\prime}} H=\left(y_{S_{f}+1} \cdots y_{i} \cdots y_{2 S_{f}}\right)^{\prime} \\
\text { with } & : y_{i}=X_{i}{ }^{\prime} H .
\end{aligned}
$$

The matrices $A^{(k)}$ should take into account the neighbouring chips over a size as large as the filter, for obtaining an accurate model of the signal to be filtered. It is important to note that one has to average the calculated BER of each possibility of the chip configuration around a bit. Obviously, the higher the filter order is, the higher the number of neighbouring chip configurations are.

\section{B. Non Coherent Demodulation}

The sign of the difference between the sum of the chip samples should be studied. Thus a detection of the bit equal to ' 1 ' occurs when:

$$
Y^{(1)^{\prime}} \Lambda Y^{(1)}>Y^{(2)^{\prime}} \Lambda Y^{(2)}
$$

where $\Lambda=\operatorname{diag}\left(\alpha_{1}, \cdots, \alpha_{S_{f}}\right)$ is the filtering matrix used for the bit decoding. In this paper, the case where $\Lambda=I_{S_{f}}$ is

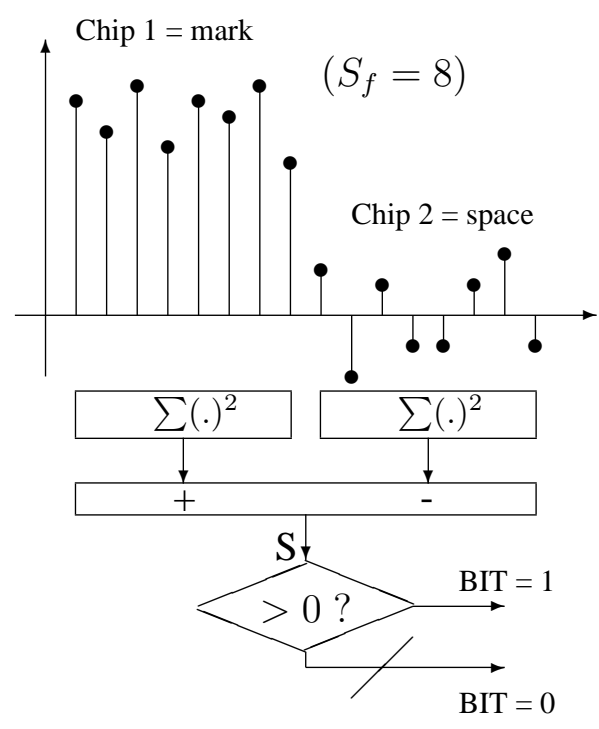

Fig. 4

BI-PHASE NON COHERENT BIT DECODING

studied. In the future work, mentioned in Section $\mathrm{V}, \Lambda$ should be set to its optimum value, depending on the filter $H$ in order to match the filtered signal. In other words, the sum over each chip could be balanced so as to favour the reliable samples thereby increasing the performance. Equation (10) becomes:

$$
\left(\sum_{i=1}^{S_{f}} y_{i}{ }^{2}-\sum_{i=S_{f}+1}^{2 S_{f}} y_{i}^{2}\right)>0 .
$$

If the bit is '0', swapping indexes gives the same equation. Fig. 4 shows how to decode a bit.

Since $Y^{(k)}, k \in\{1,2\}$ is a linear combination of gaussian probability density functions (PDFs), it has also a gaussian PDF.

Let $Y^{(k)}$ have a mean value $\mu^{(k)}=E\left(Y^{(k)}\right)$ and a covariance matrix $V=\operatorname{Cov}\left(Y^{(k)^{\prime}}, Y^{(k)}\right)$. We have:

$$
\begin{aligned}
\mu^{(k)} & =E\left(Y^{(k)}\right)=E\left(X^{(k)^{\prime}} H\right) \\
& =E\left(A^{(k)^{\prime}} H+B^{(k)^{\prime}} H\right)=A^{(k)^{\prime}} H,
\end{aligned}
$$

since $B_{(k)}$ is a random matrix whereas $A_{(k)}$ and $H$ are not. We have also:

$$
\begin{aligned}
& V=\operatorname{Cov}\left(Y^{(k)^{\prime}}, Y^{(k)}\right) \\
& =E\left(\left(Y^{(k)}-E\left(Y^{(k)}\right)\right)^{\prime}\left(Y^{(k)}-E\left(Y^{(k)}\right)\right)\right) \\
& =E\left(H^{\prime} B^{(k)} B^{(k)^{\prime}} H\right)=\left(\nu_{i, j}\right)_{1<i<S_{f}} \\
& 1<j<S_{f} \\
& \text { where } \nu_{i, j}=\sum_{m=1}^{N} \sum_{n=1}^{N} h_{m} h_{n} E\left(b_{m, i} b_{n, j}\right) \\
& \text { with } E\left(b_{m, i} b_{n, j}\right)=0 \text {, if } b_{m, i} \neq b_{n, j} \\
& =\sigma^{2}, \text { if } b_{m, i}=b_{n, j}
\end{aligned}
$$




$$
\text { and } b_{m, i}=b_{n, j} \text { when } m-n=(j-i) \Delta
$$

So $V$ is a $S_{f} \times S_{f}$ squared Toeplitz matrix because $\nu_{i, j}$ is only function of $(j-i)$; moreover it is symmetrical because $\nu_{i, j}=\nu_{j, i}$. Finally, it is identical for $Y^{(1)}$ and $Y^{(2)}$ because it only depends on the noise and the filter.

\section{Bit ERror Rate CAlculation}

\section{A. Quadratic Form Diagonalization}

The bit error rate is the probability to obtain:

$$
\operatorname{Pr}(Q<0) \triangleq \operatorname{Pr}\left(Y^{(1)^{\prime}} Y^{(1)}-Y^{(2)^{\prime}} Y^{(2)}<0\right) .
$$

So the probability density function (PDF) of $Y^{(1)^{\prime}} Y^{(1)}$ $Y^{(2)^{\prime}} Y^{(2)}$ should be calculated. The filtering introduces correlation between samples making the calculation of (21) not trivial. Quadratic form diagonalizations (well described in [4]) have to be performed taking into account the correlation factors between filtered samples.

Definitions of quadratic forms: Let the random vector $Y^{(1)}=\left(Y_{1}, \cdots, Y_{S_{f}}\right)^{\prime}$ have the mean value $\mu^{(1)}=$ $E\left(Y^{(1)}\right)=\left(\mu_{1}, \cdots, \mu_{S_{f}}\right)^{\prime}$ and the positive definite covariance matrix $V=E\left(\left(Y^{(1)}-\mu^{(1)}\right)\left(Y^{(1)}-\mu^{(1)}\right)^{\prime}\right)=\operatorname{Cov}\left(Y^{(1)}\right)$. The quadratic form in the random variables $Y_{1}, \cdots, Y_{S_{f}}$ associated with an $S_{f} \times S_{f}$ symetric matrix $\Omega$ is defined as:

$$
\Gamma\left(Y^{(1)}\right)=Y^{(1)^{\prime}} \Omega Y^{(1)} .
$$

We will now focus our attention on the particular case where $V$ is positive definite $(V>0)$.

Equation (21) is the difference between two quadratic forms defined by:

$$
G\left(Y^{(k)}\right)=Y^{(k)^{\prime}} Y^{(k)}, k \in\{1,2\}
$$

in the particular case where $\Omega=I_{S_{f}}$. So:

$$
G\left(Y^{(k)}\right)=\left.\Gamma\left(Y^{(k)}\right)\right|_{\Omega=I_{S_{f}}} .
$$

Normalisation: Let $Z^{(k)}=V^{-\frac{1}{2}}\left(Y^{(k)}-\mu^{(k)}\right), E\left(Z^{(k)}\right)=$ 0 and $\operatorname{Cov}\left(Z^{(k)}\right)=I_{S_{f}}$. Then (23) becomes:

$$
G\left(Y^{(k)}\right)=\left(Z^{(k)}+V^{-\frac{1}{2}} \mu^{(k)}\right)^{\prime} V\left(Z^{(k)}+V^{-\frac{1}{2}} \mu^{(k)}\right)
$$

Diagonalization: Let $P$ be the orthogonal matrix which diagonalizes $V$, i.e.:

$$
P^{\prime} V P=\operatorname{diag}\left(\lambda_{1}, \cdots, \lambda_{S_{f}}\right), P P^{\prime}=I_{S_{f}}
$$

Let $U^{(k)}=P^{\prime} Z^{(k)}$ with $E\left(U^{(k)}\right)=0$ and $\operatorname{Cov}\left(U^{(k)}\right)=I_{n}$, and let $b^{(k)}=P^{\prime} V^{\frac{1}{2}} \mu^{(k)}=\left(b^{(k)}{ }_{1}, \cdots, b_{n}^{(k)}\right)^{\prime}$. Then (25) becomes:

$$
\begin{aligned}
G\left(Y^{(k)}\right) & =\left(U^{(k)}+b^{(k)}\right)^{\prime} P^{\prime} V P\left(U^{(k)}+b^{(k)}\right)(27) \\
& =\sum_{j=1}^{S_{f}} \lambda_{j}\left(U_{j}^{(k)}+b^{(k)}{ }_{j}\right)^{2}
\end{aligned}
$$

Thus, if:

$$
Y^{(k)} \sim N_{S_{f}}\left(\mu^{(k)}, V\right), V>0,
$$

Equation (23) can be expressed as a linear combination of independent non central chi-square laws - denoted $\mathcal{X}^{2}$ laws with one degree of freedom.

The error probability per bit is given by:

$$
\begin{gathered}
\operatorname{Pr}\left(G\left(Y^{(1)}\right)<G\left(Y^{(2)}\right)\right)= \\
\operatorname{Pr}\left(\sum_{j=1}^{S_{f}} \lambda_{j}\left(U_{j}^{(1)}+b^{(1)}\right)_{j}^{2}<\right. \\
\left.\sum_{j=1}^{S_{f}} \lambda_{j}\left(U^{(2)}{ }_{j}+b^{(2)}{ }_{j}\right)^{2}\right)
\end{gathered}
$$

which can be written with shorter notations, assuming that:

- for $1 \leq j \leq S_{f}$, the indexed variable refers to the first chip

- for $S_{f+1} \leq j \leq 2 S_{f}$, the indexed variable refers to the second chip:

$$
\operatorname{Pr}(Q<0)=\operatorname{Pr}\left(\sum_{j=1}^{2 S_{f}} \lambda_{j}\left(U_{j}+b_{j}\right)^{2}<0\right),
$$

with:

$$
\begin{aligned}
& \left(\lambda_{j}\right)_{1<j<2 S_{f}}=\left(\lambda_{1}, \cdots, \lambda_{S_{f}},-\lambda_{1}, \cdots,-\lambda_{S_{f}}\right) \\
& \left(\lambda_{1}, \cdots, \lambda_{S_{f}}\right) \text { being the } S_{f} \text { eigenvalues of } V \\
& U_{j}: \text { central normal random variable } \\
& \left(b_{1}, \cdots, b_{2 n}\right)^{\prime}=\left(b_{1}^{(1)}, \cdots, b_{S_{f}}^{(1)}, b_{1}^{(2)}, \cdots, b_{S_{f}}^{(2)}\right)^{\prime} \\
& \left(b^{(k)}, \cdots, b^{(k)}{ }_{S_{f}}\right)=P^{\prime} V^{-\frac{1}{2}} \mu^{(k)} .
\end{aligned}
$$

\section{B. Bit Error Rate Evaluation}

The problem is now solved by evaluating the PDF of $Q$ which is a linear combination of non central $\mathcal{X}^{2}$ laws (31). The non central $\mathcal{X}^{2}$ laws are assumed to be independent, which is

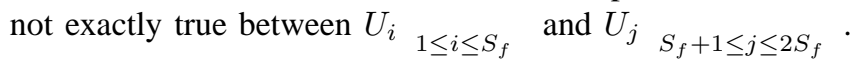
In fact independence does exist inside each chip, but not between different chips.

Two evaluations have been considered, both of them described by J.P. Imhof [5]: the first one is based on an approximation of the law of $Q$ and the second one is a numerical inversion of the characteristic function of $Q$.

1) $\mathcal{X}^{2}$ Distribution Approximation of $Q$ : Let the distribution of $Q$ be approximated by that of $c \mathcal{X}^{2}(h, 0)+b$, where $c$, $h$ et $b$ are chosen so that the two distributions have the same first three moments. We have then:

$$
\operatorname{Pr}(Q<0) \simeq \operatorname{Pr}\left(\mathcal{X}^{2}(h, 0)<y\right)
$$

where:

$$
h=\frac{c_{2}{ }^{3}}{c_{3}{ }^{2}}, y=-c_{1} \sqrt{\frac{h}{c_{2}}}+h, c_{i}=\sum_{j=1}^{2 S_{f}} \lambda_{j}{ }^{i}\left(1+i b_{j}{ }^{2}\right) \text {. }
$$

In the case where $Q<0$ and $c_{3}<0$ the distribution of $-Q$ should be approximated and $\operatorname{Pr}(-Q>0)$ should be evaluated. 
2) Numerical Integration Technique: With the approximation that the $2 S_{f}$ elements of the sum (31) are independent, the characteristic function of $Q$ is given by [5]:

$$
\phi(t)=\prod_{k=1}^{2 S_{f}}\left(1-2 i \lambda_{k} t\right)^{-\frac{1}{2}} \exp \left(i \frac{b_{k}{ }^{2} \lambda_{k} t}{1-2 i \lambda_{k} t}\right)
$$

We have ([6], [7])

$$
\operatorname{Pr}(Q<0)=\frac{1}{2}-\frac{1}{\pi} \int_{0}^{+\infty} \frac{\sin \theta(u)}{u \rho(u)} d u
$$

with:

$$
\begin{aligned}
& \theta(u)=\frac{1}{2} \sum_{r=1}^{2 S_{f}} \arctan \left(\lambda_{r} u\right)+b_{r}{ }^{2} \lambda_{r} u\left(1+\lambda_{r}{ }^{2} u^{2}\right)^{-1}(36) \\
& \left.\rho(u)=\prod_{r=1}^{2 S_{f}}\left(1+\lambda_{r}{ }^{2} u^{2}\right)^{\frac{1}{4}} \exp \frac{1}{2} \frac{\left(b_{r} \lambda_{r} u\right)^{2}}{\left(1+\lambda_{r}{ }^{2} u^{2}\right)}\right)
\end{aligned}
$$

The function $u \rho(u)$ increases monotonically toward $+\infty$. Therefore numerical integration can be carried out over a finite range.

\section{RESUlts}

A comparison between the theoretical BER and the simulated transmission BER, which gives very good results, is presented in the first part of this section. With this new reliable tools, the influence of all the parameters governing the transmission can be easily determined, thus allowing the optimization of a given system. The main parameters which have been studied are:

- Decimation factor

- Number of filter coefficients

- Type of filter design

- Offset between decimated and non-decimated signal

The second and third part of this section will present the influence of two of them. The default value of the transmission parameters used are listed below:

- $2 S_{f}=1024$ samples per bit

- low pass filter with $N=512$ coefficients

- decimation factor $\Delta=128$

The bit error rates are shown in this paper as a function of the signal to noise ratios (SNRs), defined as:

$$
S N R=\frac{U^{2}}{2 \sigma^{2}},
$$

$U$ and $\sigma$ being defined in II-A.

\section{A. Reference Results}

Fig. 5 shows the BER obtained with the default parameters. Both filtered and non filtered transmission BER are shown. For the filtered case, both methods appear: the approximate one (III-B.1) and the numerical one (III-B.2). Our numerical model is very satisfactory since it fits the simulated BER and is much faster to compute than the simulations. Although the approximate model differs slightly from the simulation, it is yet a good approximation and remains quite useful for rough optimizations since it is faster to calculate than the numerical integration (Tab. I). The filtering process seems to decrease the BER; this point is discussed in the next section.

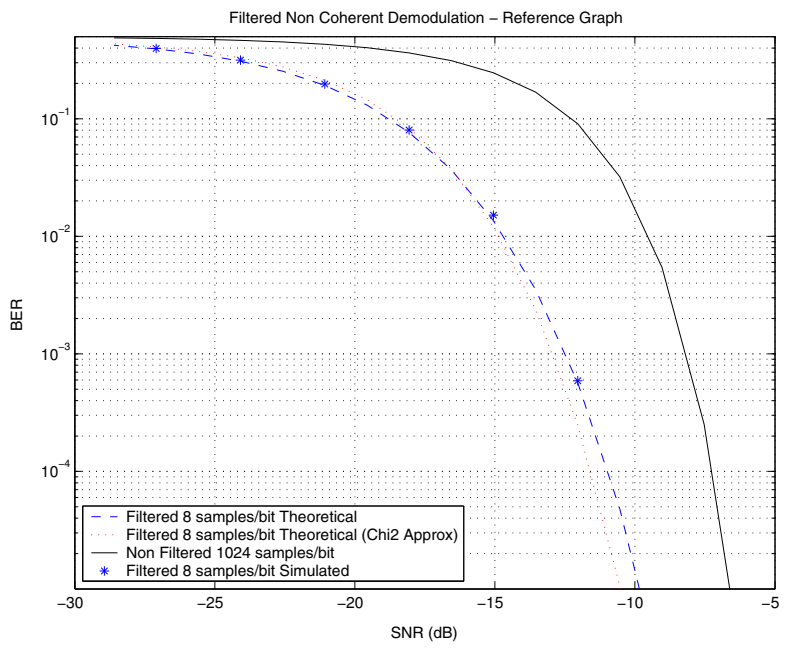

Fig. 5

REFERENCE BER

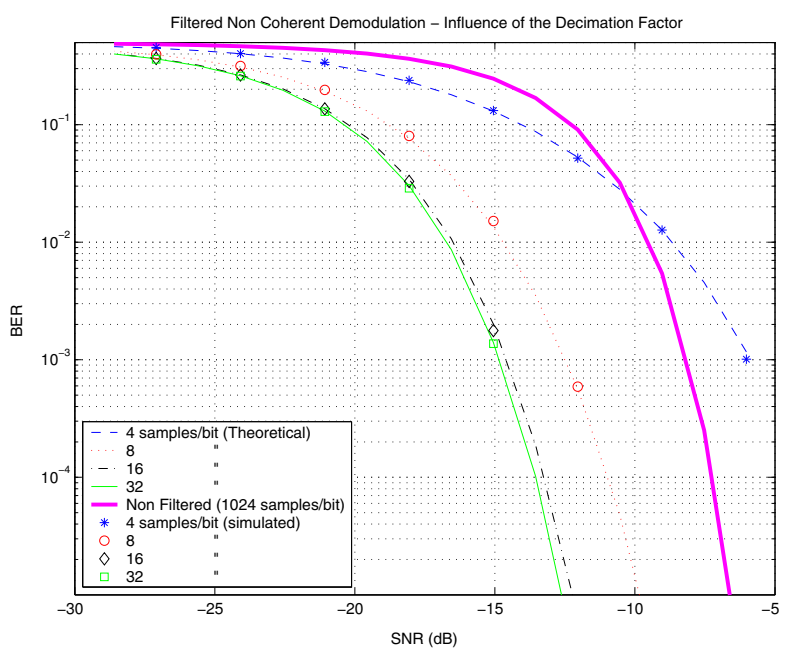

Fig. 6

DECIMATION FACTOR INFLUENCE

\section{B. Influence of the Decimation Factor}

Fig. 6 shows the influence of the decimation factor. As expected, the higher the decimation factor is, the worse the performance is.

We can observe that for the low SNRs, filtering decreases the BER. But for the high SNRs, filtering increases the BER. In fact, filtering has two opposite effects on the non coherent demodulation:

- filtering smoothes the signal, and thus decreases the contrast between mark and space. This effect tends to increase the BER.

- filtering reduces the noise band, increasing the SNR and thus decreasing the BER. 
TABLE I

CALCULATION TIME RANGE COMPARISON

\begin{tabular}{|l||c|c|c|}
\hline Evaluation method: & Simulation & Numerical integration & $\mathcal{X}^{2}$ approximation \\
\hline Time range: & 8 hours & $\simeq 20$ seconds & $\simeq 5$ seconds \\
\hline Note: & $\begin{array}{l}\text { Work has been performed using MATLAB on a } 1 \mathrm{GHz} \text { Pentium III based personal computer } \\
\text { featuring } 512 \mathrm{Mb} \text { RAM and running under Microsoft Windows 2000. Simulations have been } \\
\text { computed over } 10^{5} \text { bits. }\end{array}$
\end{tabular}

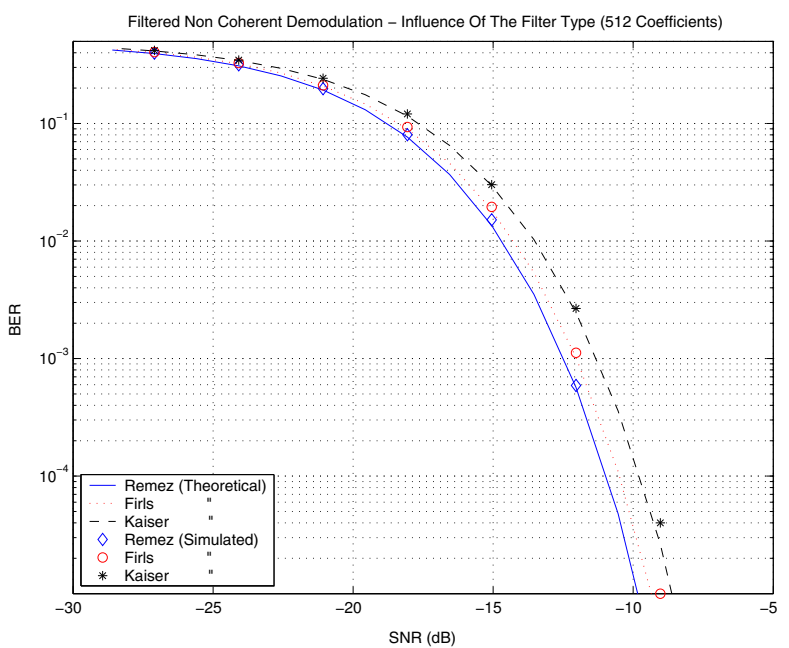

Fig. 7

FILTER DESIGN INFLUENCE

\section{Influence of the Filter Design}

With given filter specifications, the choice of a digital filter type is often based on its number of coefficients and their dynamic. But these different types of filter designs (Remez or Kaiser design for example) do not have the same influence on the BER.

Fig. 7 shows three BERs obtained with three different filter designs respecting the same frequency specifications; we can clearly see that the BER argument can make the difference in the choice of a design technique.

\section{CONCLUSION}

In this paper, we have proposed a mathematical expression for the bit error rate in the case of a multiband OOK non coherent demodulation. The OOK modulation is very common in instrumentation and measurement where the costs and the consumption are very important constraints. This method is based on a theoretical study using linear combinations of chisquare laws. The expression obtained is the exact bit error rate of the transmission and takes into account each parameter of the transmission, including the digital filtering parameters. The BER obtained fits perfectly the simulations results. This method is very fast compared to simulations of the system. Its major advantage is that optimization of the transmission parameters is much easier to perform in terms of bit error rate.
The present work will be extended to the more general case where the filter has complex coefficients, and the influence of a shift between the signal and the filter bandpass will be analized. We may also consider the use of a match filter before decoding, so as to favour the reliable samples thereby increasing the performance.

\section{REFERENCES}

[1] J. Proakis, Digital Communications. McGraw-Hill International, third ed., 1995.

[2] M. Schwartz, W. Bennett, and S. Stein, Communication Systems and Techniques. McGraw-Hill New-York, 1966.

[3] E. Boutillon, J. Danger, L. Fonseca, A. Garcia, and L. Gonzales, "Digital demodulator matching on a fpga," in Workshop on Signal Processing Systems, (Boston), IEEE, 1998.

[4] A. Mathai and S. Provost, Quadratic Forms In Random Variables : Theory and Applications. Marcel Dekker, Inc., 1992.

[5] J. Imhof, "Computing the distribution of quadratic forms in normal variables," Biometrika, vol. 48, pp. 419-426, 1961.

[6] J. Gil-Pelaez, "Inversion formulae for the distribution of ratios," Ann. Math. Statist., vol. 19, pp. 228-37, 1948.

[7] J. Gurland, "Note on the inversion theorem," Biometrika, vol. 38, pp. 481$2,1951$. 